\title{
A (2). OVERALL STRUGTURE: \\ SPACE DISTRIBUTION AND MOTIONS IN THE DISK
}

The population of the disk consists of a great variety of objects with a wide range of concentration towards the galactic plane and of peculiar motions. The extreme population I objects like interstellar gas and dust and supergiant stars, show quite different properties with respect to space distribution and motions, compared to such stars as, for instance, the common G and $\mathrm{K}$ giants. The discussions at the conference have concentrated on the observational possibilities of studying the correlation-or the lack of correlation-between the space distributions of different kinds of objects. From recent theoretical as well as observational work it has become apparent that there is a wide range in the ages of the stars contributing to the disk population, from a few million so some rooo million years. The differences in the distributions of the various kinds of stars very probably must be interpreted in terms of these differences in age and evolution. The most intriguing problem for the present thus seems to be this: Can we trace large-scale structure in the distribution of objects of different ages, and can we derive information on the evolution of the galactic system and of the stars themselves from the degree of resemblance in the large-scale structure exhibited by different objects?

Of great importance in this study is the comparison between our Galaxy and other stellar systems, particularly the nearest spiral nebulae. From these objects we derive information on the distribution of the most luminous stars of various types and of the interstellar medium and on their mutual relation. It is, therefore, appropriate first to summarize some of the results of the studies in this field communicated by Baade.

\section{INFORMATION DERIVED FROM THE ANDROMEDA NEBULA}

The Andromeda nebula is particularly suited for study of the properties of spiral arm and inter-arm populations, because it exhibits a well-defined spiral structure. Its arms are relatively thin and secondary branches which so easily confuse the picture are rare. Evidence of heavy absorption in the arms was derived from the fact that on $\mathrm{H}_{\alpha}$ photographs emission nebulae appear by the hundreds in the arms-and the brightest and largest among 
them appeared also on the red plates used for the resolution of the general background, whereas none of them showed up on the earlier plates surveyed by Hubble. This indicated heavy selective absorption because the spectra of these emission nebulae differ in no way from those of the emission nebulosities of our own Galaxy.

On the other hand, the regions between the arms seem to be free from heavy absorption, as appears from the lack of reddening of globular clusters shining through them and from the observation of distant extragalactic nebulae through the inter-arm medium. Thus, the dust appears to concentrate in the arms. There are theoretical reasons to believe that dust and gas have the same distribution, and since in our Galaxy the spiral structure of the gas has been clearly demonstrated by the recent observations of the $21 \mathrm{~cm}$. hydrogen emission line, we assume that in the Andromeda nebula too, the gas is concentrated with the dust in the spiral arms. Though, as was pointed out by Lindblad, there are some striking deviations of the lanes of dark matter of lower intensity in the inner region from the general spiral pattern.

The spiral arms of gas and dust are also the regions where the supergiants appear. The question rises whether gas and dust are primary and the stars secondary or whether the stars are primary and produce the gas and dust. We adopt the first alternative because:

(a) The short lifetimes of the super-giants require that these stars are continuously replenished and that star-formation is continuously going on in the dust and gas. There are reasons to believe that together with the super-giants, stars of all sorts of masses down to faint dwarfs are being formed in the associations.

(b) If gas and dust are the primary features of spiral structure, systems, otherwise similar to $\mathrm{M}_{3} \mathrm{I}$, but free of gas and dust, should be unable to develop spiral structure and no super-giants should appear. Such systems actually occur: the So nebulae, where the absence of gas may be due to collisions with other galaxies.

The stars observed in the central regions, the inter-arm region and the outermost regions, which are mainly population II objects (the resolution in these parts occurs simultaneously with the resolution of the globular clusters in $\mathrm{M}_{3} \mathrm{I}$ ) must be considered as disk population according to the shapes of the isophotes as measured, for example, by Hiltner and Williams. The disk population of type II seems to be the main source of light in the photometry of the nebula. Holmberg ${ }^{1}$ found for the Andromeda nebula (NGG 224) the integrated colour index $C I=+0.86$ which is very close to the mean value +0.88 found by Stebbins and Whitford for the nearer 
elliptical nebulae. ${ }^{1}$ The spiral arms with their population I stars are imbedded in this population II disk. However, it probably would be going too far to state that all the inter-arm stars are population II, because the spiral structure must have added new stars ever since the first disk stars were formed. Some of the newly formed stars will diffuse from the spiral arms into the inter-arm regions, and we should expect to find there a mixture of stars of all ages. The real composition of the spiral arm and inter-arm population in terms of populations I and II or in more refined classification into stars of various sub-systems or ages, is a problem that can be studied only in our own Galaxy.

Another result which is indicated by the study of the Andromeda nebula should be mentioned; from the surveys of the Cepheids and the OB supergiants it appears that both these objects occur in the spiral arms. But a high frequency of $O B$ super-giants in a certain section of a spiral arm does not necessarily mean a concurrent high frequency of the Cepheids. What seems to be correlated is the frequency of the F, G and $\mathrm{K}$ super-giants and that of the Cepheids. For a final verdict three colour-measures are required to eliminate the ever-present reddening effects.

\section{DISTRIBUTION AND MOTION OF INTERSTELLAR GAS}

By far the most complete information on the distribution of the interstellar gas in our Galaxy is that being derived from the $21 \mathrm{~cm}$. emission line of neutral hydrogen. It is based on measures of the Doppler shift, combined with an assumption concerning the rotational velocities in different parts of the Galaxy. The rotational velocities adopted up to now are based on the supposition that the mean motion of the gas is everywhere circular and shows rotational symmetry with respect to the nucleus. At distances from the centre equal to that of the Sun, the change of the velocity of rotation with the distance from the centre is immediately derived from the constants of galactic rotation and hence from proper motions and radial velocities of stars. At smaller distances from the centre this dependence can be derived, at least partly, from the radio measures themselves; for the outer parts of the Galaxy the radio measures will not provide such direct information and the interpretation of the measures depends largely on a model of our Galaxy. For recent results dealing with the distribution of the neutral interstellar hydrogen, we refer to an article by Van de Hulst, Muller and Oort. ${ }^{2}$

Measures of the continuous radiation at decimetre wave-lengths will furnish information on the distribution of ionized hydrogen. 
Possible deviations of the mean motion of the gas from the direction of circular motion, and from rotational symmetry, can in general be detected only when mean motions and distances are measured for distant stars which are closely associated with the gas. Such are the super-giant stars and to the best present knowledge the Cepheids of population I. The discovery of very distant $\delta$ Cephei variables and OB stars, and the photometry and measurement of radial velocities of these objects is, therefore, of the greatest importance.

\section{SUPER-GIANTS}

Recent studies of the distribution of emission nebulae and of the O-associations have revealed the spiral pattern in the distribution of these objects up to about 3000 parsecs. An article by Morgan, Whitford and Code in the Astrophysical Journal, 118, No. 2, 1953, gives the results described by W. W. Morgan at the meeting. Preliminary results of a survey of the southern emission nebulae by Bok, Bester and Wade were presented by Bok; this survey clearly shows the inner spiral arm running through the Carina concentration of early type stars. The distribution of the southern O-associations is being studied by Code from observations collected at the Radcliffe Observatory. The evidence available at present indicates that the spiral structure of the luminous $O$ and $B$ stars coincides with that exhibited by the neutral hydrogen.

We have no clear-cut information yet how the distribution of $\mathrm{A}$ and $\mathrm{F}$ type super-giants compares with that of the $\mathrm{O}$-associations. In the nearby spirals the $\mathrm{O}, \mathrm{B}$ and later type super-giants are intermingled in the spiral arms, but the percentage with which the various types are represented varies from place to place. Similar variations in our own system will have to be studied by means of accurate spectral classifications and photometry, using the finding lists of O-F super-giants published by the Tonantzintla, Warner and Swasey, and Yerkes Observatories. ${ }^{1}$ The finding lists published up to now have a limiting magnitude of about Io, and extension to the I2th magnitude, which obviously is very important, is under way at the Tonantzintla Observatory. Particular emphasis, according to W. W. Morgan, should be laid on the homogeneity of the systems used for the spectral classification and the photometry, and it is strongly recommended that for the latter the UBV system, ${ }^{2}$ applied in conjunction with the revised Yerkes system of spectral classification, be adopted. Further planning of these programmes was not considered desirable at the conference, as the observatories which are in a position to carry them out are already active in this field. 
The problem of finding the $\mathrm{OB}$ stars fainter than the limits of the above-mentioned spectral survey was the subject of extensive discussions at the conference. Searching for very faint Hir regions, as has been done with the 48-inch Schmidt, is successful in so far as it reveals those OB stars which are associated with the interstellar gas, but on account of the strong reddening it is often difficult to identify the exciting star (experience reported by Baade). Other methods which might be feasible, suggested by W. W. Morgan, are:

(a) Surveys of stars showing hydrogen emission lines, as carried out by Merrill and associates. ${ }^{1}$ Their last catalogue, which lists early type stars showing $\mathrm{H}_{\alpha}$ emission, is a suitable basis for the selection of some of the distant high luminosity OB stars. The objects listed belong to two categories of stars which happen to be well separated in luminosity, on the one hand low luminosity main sequence $\mathrm{Bo}^{-\mathrm{B}_{5}}$ stars, with a range in absolute magnitude around -3 to $-I$ and, on the other, super-giants with luminosities around -6 or -7 . From a plot of all the stars in this last list (which are rather fainter than those in the earlier lists) it appears that in certain ranges of galactic longitude the faint objects, which are about I I th magnitude, are strongly concentrated to the galactic equator. These very probably are distant and hence very luminous objects and their further observation might reveal a large fraction of super-giants. This method could in principle be extended to fainter stars. Experience at the Tonantzintla Observatory shows that emission stars up to the $14^{\text {th }}$ magnitude can be found; actually, considerable numbers of new emission objects have been found already in a region centred at NGG $663 .{ }^{2}$ The repetition of Merrill's survey up to $m=14$ is under consideration at Tonantzintla.

(b) Multi-colour photographic photometry might be used as a first step in sorting out the objects among which the luminous B-stars are to be found. As a next step accurate photo-electric multi-colour photometry could then pick out the objects sought. This procedure would rapidly converge and would be not too time-consuming.

That the criteria in searching for the O-F super-giants on the Schmidt objective prism plates at Cleveland and Tonantzintla have been successful in revealing a high percentage of real super-giants, has been confirmed by the observations with slit spectra. The situation is more difficult for types $\mathrm{G}_{2}$ and later. It turned out that the intensity of the $\mathrm{CN}$ band as a criterion for luminosity, which as a rule is a useful means of identifying the stars, is not reliable in all cases. No other reliable criterion has been found.

In addition to the photometry and accurate spectral classification of the 
stars found in the survey, determination of the radial velocities was strongly recommended. As has been stated already in connexion with the discussion of the $21 \mathrm{~cm}$. radio measures, knowledge of the mean motions in different parts of the galactic system can be determined only from the radial velocities of distant objects, and the super-giants, with their low peculiar motions are best suited for this purpose. The problem of the determination of radial velocities for these faint objects is a difficult one, but the newly developed technique of objective prism radial velocities may well prove to be the solution (see also p. 4I). The importance of such work can hardly be stressed too much.

\section{CEP HEIDS}

Cepheids can be identified at larger distances than O-F super-giants as no spectra are required. Therefore, extension of the existing surveys of Cepheids along the galactic equator to the faintest possible limits will be extremely valuable. ${ }^{1}$ The survey should be supplemented with colour measures and, for the brighter objects, with observations of the radial velocities. It is particularly from the radial velocities of Cepheids that we may derive information about the velocity of rotation around the galactic centre as a function of the distance from the centre. The early work on radial velocities by Joy is now being extended to the southern hemisphere by Stibbs at the Radcliffe Observatory in Pretoria, but more remains to be done.

As had been pointed out, amongst others, by Kukarkin, the present surveys are still far from complete; for instance, new Cepheids have been found by him in some regions which hitherto seemed void of these objects. This was done in a survey of selected regions which reaches up to the I 7 th magnitude. The study of the distribution of the faint Cepheids is somewhat complicated by the fact that we have a mixture of variables of populations I and II, which can be separated by the shape of the light curves only if the periods are around $15^{-20}$ days, whereas separation is very difficult for periods of a few days only. However, for the studies of the most distant regions the large periods are obviously the most important ones.

In order to penetrate to very large distances, that is to reach the faintest Cepheids in the most transparent regions, the existing surveys (Harvard, Leiden, Sonnenberg) would have to be extended to fainter limiting magnitudes in the directions of low absorption. For the selection of the most transparent regions observations with the 48-inch Palomar Schmidt will be most valuable. Also recommended is a search for the brighter 
Cepheids in the unexhausted areas for which many plates have accumulated already. Preliminary results from a survey of Cepheids in the Andromeda nebulae show that their distribution follows the spiral arms but is far from uniform. Similar conditions in the Galaxy are indicated by the crowding of Cepheids in particular regions like the Carina section. An important aim of the surveys must be to locate these rich distant concentrations.

The work on Gepheids appears to require much co-ordination, both in the planning of the faint survey and in the solution of the problem of measuring colour excesses. The latter is two-fold: how do we obtain intrinsic colours, and how will the colours of very faint objects be measured?

\section{Fields to search for faint Cepheids}

According to Baade, the selection of regions for faint extensions of the existing surveys will require very careful inspection of the 48-inch sky survey plates. Experience acquired in some provisional searching has shown that one gets occasionally, at a very low latitude, areas of remarkable transparency. A striking case is, for instance, the region around NGC 2158 , a small distant cluster in the anti-centre region. Many extra-galactic nebulae appear here on 200-inch plates. Another field is the one of the strong Cygnus A radio source, where the total absorption could be measured from the colours of the brighter E nebulae; it amounts to $2 \cdot 1$ magnitudes. Such regions will be at least as useful in outlining the outer regions of the Galaxy as the general survey of the anti-centre region (Shapley). According to Oort, the selection of regions of low absorption may be facilitated in the future when the total amount of neutral hydrogen in different directions becomes more completely known from the radio measures.

As Morgan pointed out, it has to be realized from the beginning in these faint surveys that the directions in which the Gepheids will be found to be most numerous, will be correlated with low absorption. Still, this will not seriously hamper the study of the relation between the occurrence of Cepheids and, for instance, the early type super-giants, if directions of low absorption in the nearest arm are chosen for study of the conditions in the more distant arm. Additional information on the relation between the distribution of different kinds of high luminosity stars must, of course, be anticipated from the study of the Andromeda nebula.

The matter of selecting special regions for the study of faint Cepheids was delegated by the conference to a small committee, consisting of W. Baade, B. V. Kukarkin, P. Th. Oosterhoff, and the secretary of the continuation committee on co-ordination of galactic research. This 
committee might also act in stimulating and co-ordinating the further work on the variables in these selected fields.

\section{Colours of the Cepheids}

The determination of colour excesses of distant Cepheids is a very difficult problem and this probably is the reason why, apart from Eggen's programme, ${ }^{1}$ very little has been done in this field until recently. The improvement of the photo-electric technique, however, will make possible great progress in this field in the future. A programme of photo-electric colours and magnitudes of southern Cepheids is being carried out at present by Walraven, A. B. Muller and Oosterhoff at the Leiden station in South Africa. The Cepheids are measured around maximum and the list contains all known objects brighter than $m=\mathrm{I} 4$ in maximum and south of declination $+30^{\circ}$; part of the objects are followed over the whole light curve. Another programme was started at the Boyden station but it is doubtful if this will be continued. For the northern sky no large scale programmes have been announced yet. For the interpretation of the measures it will be necessary to have accurate knowledge of the normal colours of the Cepheids. This is probably the most intricate problem of all. There are too few Gepheids close enough to the Sun to be assumed free from absorption, and indirect methods will have to be applied. The most promising one might be the application of multi-colour photometry to the foreground stars in a small field around the nearer objects, in a way outlined by Strömgren (see p. 28). This might result in a three-dimensional charting both of the stars between the Sun and the Cepheids and of the distribution of the absorbing medium. The subject requires more experimental work and its organization may be a task for the continuation committee mentioned on p. 2. Discrimination of population I and II Cepheids is an important point in all these investigations.

\section{LONG-PERIOD VARIABLES}

Another important class of variables, and one which deserves much more attention than it has received in the past, is the long-period variables. On the whole, these objects form a class intermediate between the more typical population I and II stars and they can be studied up to large distances. The dependence of their kinematical and distributional properties on the period makes them particularly interesting, ${ }^{2}$ and their large numbers allow rather detailed studies of this dependence. 
For future work, the emphasis should be in the first place on homogeneity in the discoveries. In some of the large surveys of variable stars in the past, few of the long-period variables have been discovered either because only pairs of plates with short time intervals were blinked or because the plates taken were not sufficiently uniformly distributed over a period of at least a few years to allow the determination of the period. Additional observational work may, therefore, be required. In view of the rather simple procedure of determining periods and light curves, the work on these variables would soon give valuable results.

The new survey should be combined with radial velocity and proper motion measurements for the brighter objects (see Section G).

\section{SURVEY OF OTHER VARIABLES IN THE DISK}

Interesting results on a survey of $\mathrm{T}$ Tauri stars were announced by Haro, indicating a correlation between their spectral characteristics and the physical conditions in the interstellar medium. These results have meanwhile been published in the Tonantzintla and Tacubaya Bulletin, No. 8 . Similar work for fainter stars in small regions of the dark cloud complexes is being done by Herbig at Lick. ${ }^{1}$ This work does not require further co-ordination.

\section{OPEN GLUSTERS}

The modern view on stellar evolution has a very interesting bearing on the study of open clusters. Information on the age of the clusters may be obtained from the study of their physical characteristics, and this should be combined with the study of their space distribution. As was stressed in particular by Ambartsumian, we know that the clusters which contain O-type stars are associated with the $\mathrm{O}$ associations and hence follow the spiral pattern of the $\mathrm{OB}$ stars. We would like to know the distribution of clusters in which the brightest stars are of types B or even $\mathrm{A}$, and which sometimes contain late-type giants. If these are clusters which some time in the past contained $O$ stars, we want to compare their distribution with that of the late-type giants in general in the Galaxy. More generally, from the comparison of the distribution of clusters of different physical characteristics we may derive either information concerning the evolution of the stars or concerning the evolution of the spiral pattern in the Galaxy.

The work on clusters can be divided into the search for fainter, distant clusters not listed up to now, the photometric and spectroscopic study of as many objects as is possible, and the measurement of radial velocities. 
The existing surveys by Shapley, Trumpler, and Collinder contained clusters in which the brightest stars are brighter than the 16 th magnitude. Undoubtedly the Palomar sky survey will increase the number of known open clusters.

For the distance determination of the faintest objects, where photoelectric methods would be too time consuming, the photographic threecolour method developed by $\mathrm{W}$. Becker has given very promising results. ${ }^{1}$ As was shown by Becker, the distribution of clusters can be determined in this way up to distances including the spiral arms next to the one in which the Sun is located, and a close coincidence of the seventeen clusters studied with the arms was found. If a sufficient number of stars are measured in the cluster the precision of the distance determination depends mainly on the accuracy of the zero point of the photometric scale; an accuracy of $10 \%$ in the distances had been reached in the cases shown.

Becker intends to extend this work to the southern hemisphere and to some other regions of particular interest. A large scale programme all along the galactic circle would seem very valuable. According to the experience reported by Heckmann, Schmidt cameras of moderate sizes will be most useful, with focal lengths about $1 \frac{1}{2} \mathrm{~m}$. and apertures about $40-60 \mathrm{~cm}$.; they can reach a limiting magnitude of about 19 . This might well be a very attractive programme for small observatories. The photometric system to be used requires careful consideration. The necessity of having reliable standards well spread along the Milky Way was stressed at the conference; the details about the photometry were not discussed. The matter of choosing standard sequences is, of course, to be considered in conjunction with the same problem in variable star work.

It was felt by the participants at the conference that it would be of great value if the important observations on open clusters accumulated by Dr Trumpler at the Lick Observatory during the past 20 years would become available to astronomers working in this field. The following is derived from a letter by $\mathrm{Dr}$ Trumpler of May 1953.

I. Unpublished material. Radial velocities of stars in 74 galactic star clusters divided as follows:

187 stars in the Pleiades, Praesepe, and Coma;

234 stars in 16 clusters nearer than 800 parsecs;

400 stars in 55 clusters beyond 800 parsecs.

The observations and measurements have been completed. Dr Trumpler is now occupied with a discussion of systematic errors and a reduction of the observations to a more homogeneous system based on observations of 
numerous bright standard stars. The spectral types of the stars have also been classified from the slit spectrograms. Spectral classifications from slitless spectrograms are also available for fainter stars and in other clusters, not observed for radial velocity.

2. The above mentioned data will be used for the study of galactic rotation. For this purpose distances will be re-determined using magnitudes, spectral types, and colour indices.

3. The radial velocity data will also be used for a number of subsidiary problems (spectroscopic binaries, relativity red shift, super-giants and peculiar stars in clusters, internal motions, and peculiar velocities of the clusters).

4. It is urged by $\mathrm{Dr}$ Trumpler that radial velocity observations of galactic star clusters south of $-30^{\circ}$ declination be contemplated.

The conference expressed the hope that these extensive observations will soon be published.

\section{INFRA-RED SURVEYS OF M, N, AND S STARS}

From the report given by Dr Nassau we summarize the following points which are of interest in the discussion of the population of the galactic disk. The work being done at the Warner and Swasey Observatory deals mainly with:

(a) The M-type stars, $\mathrm{M}_{5}$ and later, characterized by the presence of $\mathrm{TiO}$ bands at $\lambda$ 7054, at the telluric $\mathrm{A}$ band, and at $\lambda$ 8432. Among these stars a number show the $\mathrm{VO}$ bands and are variable. (See $A p . \mathcal{F} .119$, I 75, 1954);

(b) The $\mathrm{N}$ stars characterized by strong $\mathrm{CN}$ bands. (A list of these is published in $A p . \mathcal{F}$. 120, 129, 1954);

(c) The $\mathrm{S}$ stars recognized by the presence of $\mathrm{LaO}$ bands, and

(d) The early $\mathbf{M}$ suspected super-giants, recognized by extremely high reddening and their wedge-shaped appearance on the spectral plates; these latter stars were picked out in the present survey when they showed stronger reddening than the super-giants of this type in $h$ and $\chi$ Persei.

The first group, late $M$ stars, shows a uniformly varying density, decreasing with increasing distance from the galactic centre. The VO stars among them appear to show the same distribution as the group as a whole. Reference to the study of these stars in connexion with the density distribution in the nuclear region was already made on p. 12.

The second group, the $\mathrm{N}$ stars, appears to be of particular interest. Their distribution in the Galaxy is quite different; they show clustering, 
seem to be associated with obscurations, and show stronger galactic concentration than the late $\mathrm{M}$ giants. The number of $\mathrm{N}$ stars on which the study is based is over 300 . The counts in different directions in the galactic plane show larger numbers along the spiral arms of interstellar matter and OB stars, than outside these. Very few $\mathrm{N}$ stars were found in the directions of the Scutum and Sagittarius Clouds where the late $M$ stars are very abundant. Although they show a definite tendency to occur in groups, they do not seem to be associated with the clusterings of OB stars. They can be found up to very large distances, of the order of the dimensions of the galactic system. Further study of these stars may give very valuable results. Very little is known about their absolute magnitudes. It was suggested that one way of determining these would be to measure the intensity of the interstellar $\mathrm{D}$ lines in directions where these intensities can be calibrated by means of early-type stars. A more direct determination would be from the proper motions and radial velocities. A list of the brighter objects of this class is being compiled and will be suggested for meridian observations.

In some cases the $\mathrm{S}$ stars appear to be associated with the groupings of OB stars but it is too early to state that they belong to the same aggregates. In a number of cases the $S$ stars appear in groups. A list of $3^{1}$ new $S$ stars is in press.

The suspected early $M$ super-giants show high galactic concentration, they appear in groups and in some cases in $\mathrm{O}$ associations. A list of 89 is to be published in 1954 .

The close association of the super-giant $M$ stars with the associations of OB stars ( $h$ and $\chi$ Persei, the probable association of $\alpha$ Orionis with the Orion group) presents an evolutionary problem of great interest and should stimulate the further surveying of these stars.

Infra-red surveys of the type described by Nassau are being extended to the southern hemisphere at the Harvard Boyden Station ${ }^{1}$; this work is planned in the same way as the Cleveland Survey (covering a belt of $4^{\circ}$ width along the galactic equator). The Magellanic Clouds will also be studied. 